18. Dresdner Symposium "Herz und Gefäße - Innovationen 2012" 23.-24. November 2012 in Dresden

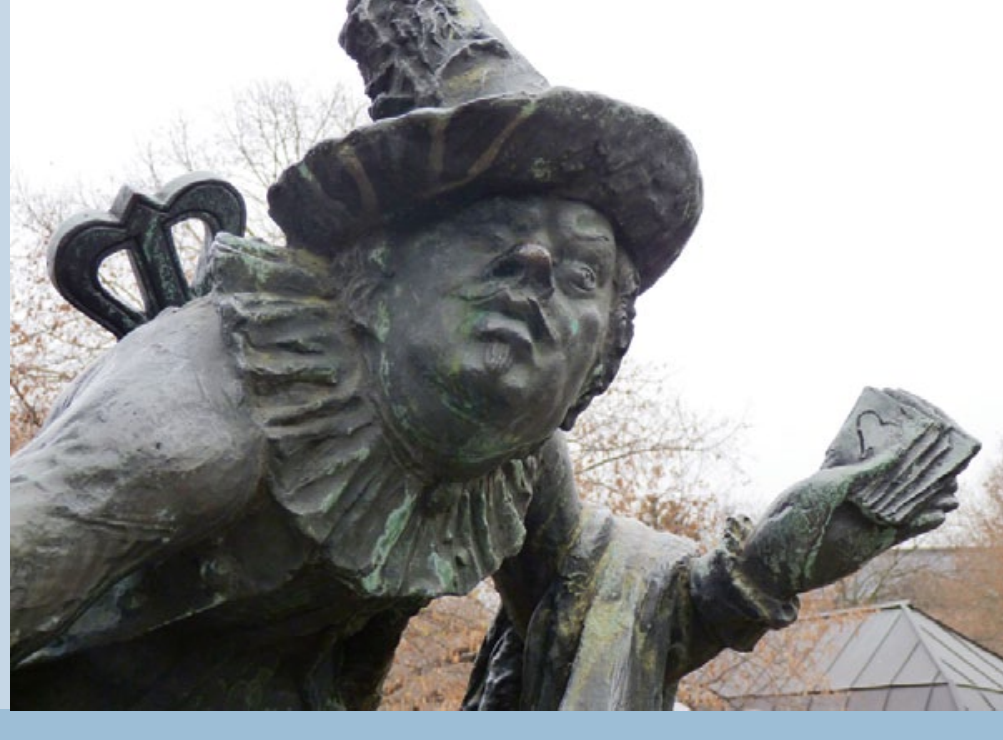

\title{
Stabile Angina Pectoris
}

\section{Alte Streitfragen, neue Studien}

\begin{abstract}
Wann konservativ, wann invasiv - um die Streitfragen zur optimalen Koronardiagnostik und -therapie definitiv zu beantworten, startet nun die weltweite ISCHEMIA-Studie. Dass nicht jede Stenose gestentet werden sollte, hatte auch die FAME-II-Studie gezeigt.
\end{abstract}

Die stabile Angina Pectoris schien sich mit der heiß diskutierten COURAGE-Studie dem interventionellen Zugriff entzogen zu haben. Denn die optimale medikamentöse Therapie schnitt mindestens ebenso gut ab. Substudien von COURAGE und auch weitere Befunde wiesen jedoch darauf hin, dass der Grad der Myokardischämie über die Prognose bestimmen dürfte. Deshalb haben die amerikanischen Gesundheitsbehörden wieder viel Geld in die Hand genommen, um endgültig eine Klärung zur „richtigen“ Koronardiagnostik und -therapie herbeizuführen: ISCHEMIA (International Study of Comparative Health Effectiveness with Medical and Invasive Approaches) heißt die weltweite Megastudie, an der auch deutsche Zentren teilnehmen.

Wie Rolf Dörr, Dresden, erläuterte, sollen 8000 Patienten an 400 internationalen Zentren randomisiert werden. Die Studienhypothese: Die initial invasive Revaskularisationsstrategie

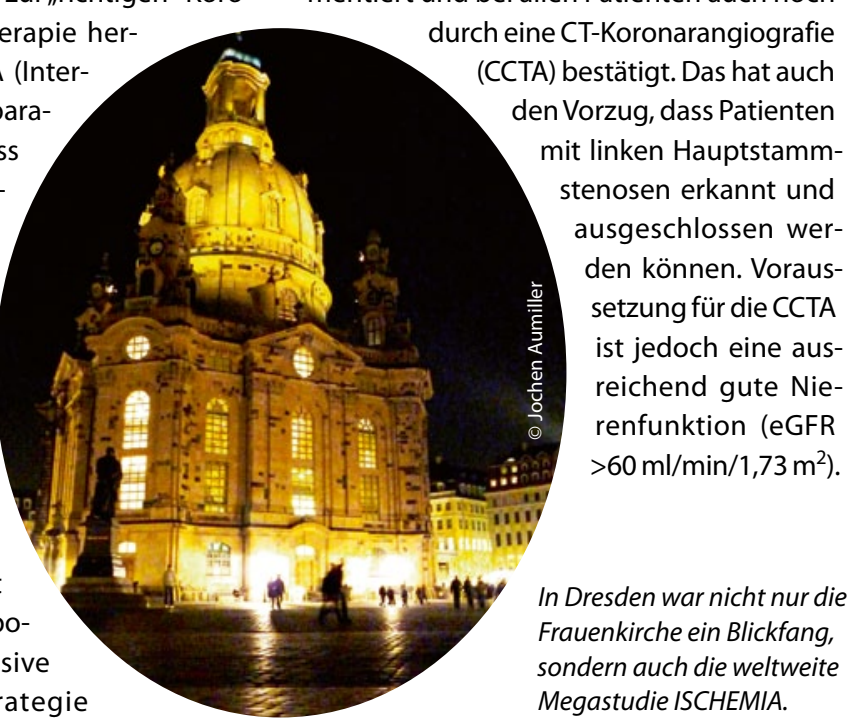

( $\mathrm{PCl}$ oder Bypass) plus optimaler medikamentöser Therapie (OMT) ist der konservativen Therapie mit OMT alleine überlegen, wenn für Komplikationen bei der OMT der Einsatz invasiver Therapieverfahren ermöglicht wird.

\section{ISCHEMIA mit maximalem Aufwand}

Stimuliert wurde ISCHEMIA durch mehrere kleinere Studien. Diese fanden im Gegensatz zu COURAGE, dass die Intervention bessere Ergebnisse erzielt, wenn ein Ischämienachweis vorausging. Sowohl in der COURAGE-, als auch in der im Ergebnis ähnlichen BARI2-Studie wurde nach der $\mathrm{PCI}$ randomisiert, was grundlegende Zweifel aufwarf. In ISCHEMIA wird der Ischämiegrad vor der Randomisierung mit den modernsten Stresstests (nuklear, Echo- oder MRT-Stresstests) dokumentiert und bei allen Patienten auch noch durch eine CT-Koronarangiografie

\section{Komplexer Studienaufbau}

Das Eingangstor für die Studie: Die KHK-Patienten müssen „alle klinischen und ischämischen Bildgebungskriterien" erfüllen. Der positive Stresstest qualifiziert für die Revaskularisation. Erscheint im Herzkatheter die Stenose größer als 50\%, kann die $\mathrm{PCl}$ erfolgen, sieht sie geringergradig aus, wird eine Messung der fraktionellen Koronarflussreserve (FFR) erforderlich. Liegt bei dieser physiologischen Druckdrahtmessung unter Adenosinbelastung (siehe Gespräch mit Prof. Werner) der Schwellenwert über 0,80, wird auf die Intervention verzichtet.

Die Bildung von Untergruppen ist auch bei negativem Stresstest vorgesehen. Zeigt die Katheteruntersuchung eine Stenose über $80 \%$, wird interveniert. Bei unter $80 \%$ ist für die Entscheidung, ob PCl ja oder nein, ebenfalls eine FFR notwendig.

Beim kombinierten primären Endpunkt beschränkt sich die Studie auf den kardiovaskulären Tod oder nicht-tödlichen Herzinfarkt. Die Liste von sekundären Endpunkten ist lang. Mit am wichtigsten die Frage: Wird durch den invasiven Therapieansatz die Lebensqualität der Patienten mit stabiler Angina im Vergleich zur alleinigen optimalen Pharmakotherapie verbessert?

Ende letzten Jahres ist die Studie angelaufen. Auf die dringend erwarteten Ergebnisse werden wir noch lange warten müssen, denn es ist eine Nachbeobachtungszeit von durchschnittlich vier Jahren vorgesehen.

Dr. med. Jochen Aumiller I 